\title{
Combination of BTrackS and Geri-Fit as a targeted approach for assessing and reducing the postural sway of older adults with high fall risk
}

This article was published in the following Dove Press journal:

Clinical Interventions in Aging

10 February 2017

Number of times this article has been viewed

\author{
Daniel J Goble \\ Mason C Hearn \\ Harsimran S Baweja \\ School of Exercise and Nutritional \\ Sciences, College of Health and \\ Human Services, San Diego State \\ University, San Diego, CA, USA
}

\begin{abstract}
Atypically high postural sway measured by a force plate is a known risk factor for falls in older adults. Further, it has been shown that small, but significant, reductions in postural sway are possible with various balance exercise interventions. In the present study, a new lowcost force-plate technology called the Balance Tracking System (BTrackS) was utilized to assess postural sway of older adults before and after 90 days of a well-established exercise program called Geri-Fit. Results showed an overall reduction in postural sway across all participants from pre- to post-intervention. However, the magnitude of effects was significantly influenced by the amount of postural sway demonstrated by individuals prior to Geri-Fit training. Specifically, more participants with atypically high postural sway pre-intervention experienced an overall postural sway reduction. These reductions experienced were typically greater than the minimum detectable change statistic for the BTrackS Balance Test. Taken together, these findings suggest that BTrackS is an effective means of identifying older adults with elevated postural sway, who are likely to benefit from Geri-Fit training to mitigate fall risk.
\end{abstract}

Keywords: aging, balance, BTrackS, Geri-Fit, postural sway, fall risk

\section{Introduction}

Recent census data show that the global, older adult population will exceed 1.6 billion individuals by the year 2050. ${ }^{1}$ Based on current trends, one-third to one-half of this population will experience a fall at least once annually. ${ }^{2}$ In light of these circumstances, it is likely that older adult falls will remain the number one cause of disability, hospitalization, and injury-related death for decades to come. ${ }^{3}$ Further, older adult falls have been linked to an increased occurrence of future falls and have been shown to underlie a "fear of falling" that dramatically reduces the quality of life. ${ }^{3}$

Given the "falls crisis" facing society, considerable efforts have been made to develop preventative screening technology that assesses fall risk. One of the more promising approaches in this domain has been the objective measurement of postural sway using center of pressure (COP) from a force plate. $\mathrm{COP}$ is the term given to the point of application of the ground reaction force vector during standing. Numerous studies have shown that increased COP measured by a force plate is indicative of poorer balance in older adults and is associated with a higher likelihood of falling. ${ }^{4-17}$

With evidence mounting in support of force plate-based fall-risk assessment, it is surprising that this preventative approach is currently not widespread. Three reasons serve as likely barriers to the mass implementation of this technology in the field. First, most force-plate devices are expensive, ranging from $\$ 5,000$ to $\$ 125,000$. Second, force 
plates typically lack portability, such that they are bolted to the ground or they are too large/heavy to easily transport. Third, force plates most often lack user-friendly software to assist in the implementation of fall-risk assessment.

In 2014, a new force plate was developed called the Balance Tracking System (BTrackS [Balance Tracking Systems Inc., San Diego, CA, USA]). This US Food and Drug Administration (US FDA)-registered, class-one medical device addresses the limitations of traditional force plates by having a low cost compared to other force plates $(\sim \$ 1,000)$, exceptional portability (lightweight; no external power), and a simple fall-risk software application (ie, BTrackS Assess Balance). These improvements are even more remarkable, based on scientific research showing that $\mathrm{BTrackS}$ is just as accurate and reliable as expensive, laboratory-grade force plates. ${ }^{18}$

Beyond fall-risk assessment, a second key component to the falls equation is determining effective balance interventions to mitigate fall risk in older adults. To this point, a recent review of the literature ${ }^{19}$ found that several exercise interventions are capable of reducing postural sway, and subsequently fall risk, in older adult participants. ${ }^{20-27}$ One limitation to this work, however, has been an inability of previous researchers to consider the baseline postural sway status of older adults prior to training. Given that postural sway is subject to ceiling effects, it would make sense that only individuals with atypically high postural sway would experience exercise-based improvements.

In the present study, an exercise intervention called Geri-Fit was implemented over 90 days in an attempt to reduce postural sway of older adults. Postural sway was measured before and after Geri-Fit training using the BTrackS Balance Plate and Assess Balance software. A comparison between individuals with lower versus higher pre-intervention postural sway was made based on the BTrackS fall-risk assessment criteria. It was hypothesized that a decrease in postural sway would be evident following Geri-Fit training and that the effects would be greatest for individuals with higher postural sway pre-intervention. This finding would serve as evidence that BTrackS is an effective means for targeting those individuals most likely to benefit from an exercise intervention like Geri-Fit for fall-risk mitigation.

\section{Materials and methods \\ Participants}

The sample for this study consisted of male $(n=6)$ and female $(n=19)$ older adults (mean \pm standard deviation: age $=74.2 \pm 5.9$ years) from the local community. Participation was voluntary and recruitment strategies included announcements in the local paper, as well as word of mouth. Participants were invited to participate if they were in good general health, and they were included because they completed all study procedures. As an incentive for undertaking the study, participants were given the associated Geri-Fit training free. Approval for this study was given by internal review board at San Diego State University and all participants provided written informed consent. Written informed consent was obtained for publication of the figure.

\section{Experimental tasks}

BTrackS Balance Test (BBT)

Balance testing in this study was conducted using the BTrackS Balance Plate and Assess Balance software as shown in Figure 1. The BTrackS Balance Plate is an FDA registered, lightweight $(<7 \mathrm{~kg})$ force plate that determines the COP of foot forces placed on it during standing with the accuracy/precision of a laboratory-grade force plate. ${ }^{18}$ The BTrackS Balance Plate surface measures $40 \mathrm{~cm}$ by $60 \mathrm{~cm}$, and the device was placed on a firm, ceramic tile surface during testing. Leveling of the board was achieved via adjustable legs on the BTrackS Balance Plate and verified with a leveling tool.

BTrackS Assess Balance software is a user-friendly computer application designed for creating user profiles and conducting tests of postural sway, for the purposes of tracking changes in COP over time and determining fall risk. The BTrackS Assess Balance software was loaded onto several laptops, which were connected to individual BTrackS Balance Plates via each plate's USB cable. This connection also provided power to each plate and allowed sampling of COP at $25 \mathrm{~Hz}$. The BTrackS sampling frequency, while lower than some other force-plate systems, was adequate, given that it satisfied the Nyquist theorem for the slow $(<10 \mathrm{~Hz})$ COP changes measured in the present study. All balance testing was performed by experienced research technicians.

The balance testing protocol used in this study was the standard protocol of the BTrackS Assess Balance Softwarethe BBT. The BBT consisted of four 20 s trials with minimal intertrial delays $(<10 \mathrm{~s})$, where each trial began and ended with an auditory tone. For each trial, the participant was instructed to stand as still as possible on the BTrackS Balance Plate with his or her eyes closed, hands on hips, and feet shoulder width apart (Figure 1). The first trial was a practice trial, while the remaining three trials were used to calculate the BBT result in centimeters. The BBT result was equal to the average, total COP path length across the three testing trials. 


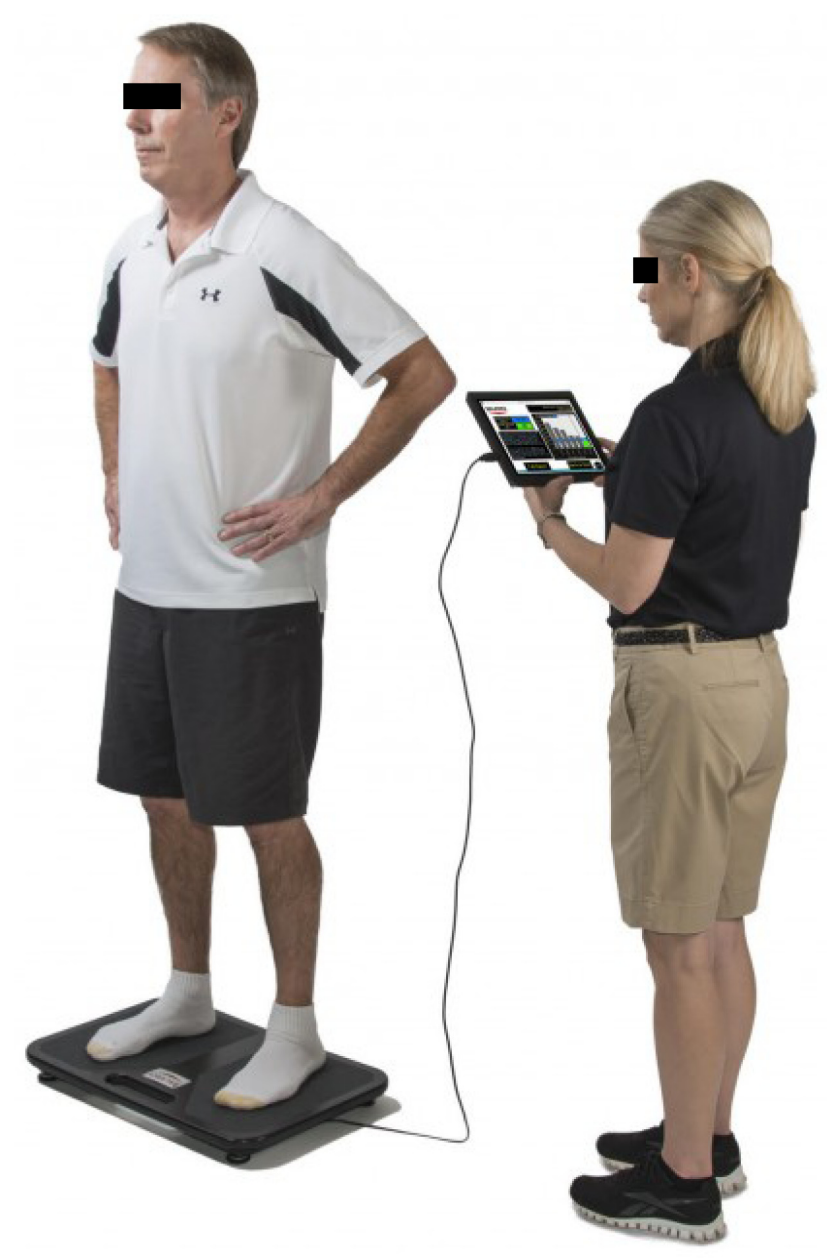

Figure I Depiction of BTrackS setup for postural sway testing. Participant stood on the BTrackS Balance Plate with feet shoulder width apart, hands on hips, and eyes closed for four $20 \mathrm{~s}$ trials. Postural sway was measured using the BTrackS Assess Balance software.

Abbreviation: BTrackS, Balance Tracking System.

\section{Geri-Fit exercise intervention}

All participants underwent the Geri-Fit exercise intervention, an evidence-based health promotion program suggested for older adults over the age of 65 years. Geri-Fit has been offered throughout the US since 1989 and, in 2012, it underwent a review process with the Administration for Community Living and the Administration on Aging. At that time, Geri-Fit was awarded the highest criteria (level III) as a disease prevention/health promotion program.

The basis of the Geri-Fit program is its group strength training classes conducted twice a week for 45 min each. Participants were required to adhere to a lesson plan that progressed over 12 weeks. All participants performed the same exercises/workouts during each class. Each class consisted of 12-min of warm-up stretching, followed by $30 \mathrm{~min}$ of progressive resistance strength training, and then 2-3 min of cool-down stretching. Most of the exercises performed were completed while sitting in a chair; however, participants had the option to do them standing up if they preferred. Most of the exercises were performed with $0.9-3.6 \mathrm{~kg}$ dumbbells depending on the age, fitness level, and the length of time the participant was enrolled in Geri-Fit. Class sizes are limited to 25 participants to assure plenty of one-on-one corrective exercise instruction is given.

For the 30 min of strength training in each Geri-Fit class, specific body parts were targeted including the back/ shoulders, legs, arms, hands, fingers, chest, and abdominals. The strength training exercises were derivatives of standard body-building exercises, such as the dumbbell row, front raise, one-arm lateral raise, one-arm overhead press, chair squats, stationary lunges, leg raises, assisted leg raises, triceps extension, dumbbell curls, hammer curls, French press, onearm triceps extension, wrist curls, forearm curls, calf raises, calf raises with holds, one-legged stands, standing superman, wall push-ups, table press-ups, seated crossovers, and side bends. In addition, one workout each month incorporated a plyometric exercise, such as the hopscotch or mini jump squats, to break up the monotony of doing the same routine each time.

As participants became more comfortable with the exercises of Geri-Fit, and subjectively showed signs of improvement, more challenging exercises were added to the workout such as overhead press, lateral raises (using both arms at once), bent over lateral raises, Arnold press, Zottman curls, concentration curls, chair dips, forward lunges, reverse lunges, and weighted squats. Progressive training techniques, ie, pulses, holds, negatives, half-reps and going to failure were employed in order to build strength in the arms and legs of older adult participants. Additionally, each participant was individually analyzed throughout each session and suggestions were made for further improvement (eg, use more weight) once a given exercise was mastered.

\section{Experimental procedure}

The postural sway of all participants was measured with the BBT before (ie, pre-intervention) and after (ie, postintervention) undergoing 90 days of Geri-Fit training. GeriFit was conducted at three local sites and participants were encouraged to make up any class they missed at another location. If this was not possible, participants used a DVD version of the program to exercise at home or, if they had computer access, they were given a link to a Geri-Fit workout available online. Attendance was high (87\%), as monitored by attendance sheets that instructors were required to keep. Those that opted to do the at-home DVD, or virtual online class when they missed the regular class, reported their exercise adherence to their instructor the next time the class met. 


\section{Data analysis}

The first-level analysis in this study determined whether a reduction in postural sway occurred from pre-intervention to post-intervention across all subjects. This analysis consisted of a paired $t$-test comparing the COP path length of the pre-intervention and post-intervention BBT results. The results of this $t$-test were considered significant at the $P<0.05$ level. Following this preliminary evaluation of the data, the participant pool was divided into two groups based on the amount of postural sway exhibited during the pre-intervention BBT.

The first group determined was the "LOWER" postural sway group, which consisted of individuals with a COP path length of $38 \mathrm{~cm}$ or less on the pre-intervention BBT. The second group was the "HIGHER" postural sway group, which consisted of those individuals with a pre-intervention COP path length of $39 \mathrm{~cm}$ and higher. These cut points were selected based on the BTrackS Assess Balance Software criteria for fall-risk categorization, whereby the LOWER group corresponded to individuals with no more than moderate risk of falling and the HIGHER group corresponded to those with high risk of falling. BTrackS determines fall risk using a large normative database of over 10,000 individuals of all ages. High fall risk (ie, COP path length of $39 \mathrm{~cm}$ and higher) represents postural sway that is greater than $98 \%$ (two standard deviations from the mean) of healthy young adults between the ages of 20 and 39 years. Two sample $t$-tests were used to compare the demographics of the LOWER and HIGHER groups.

Based on the above groupings, a second-level analysis was conducted to determine whether postural sway reductions with Geri-Fit were more evident in individuals with LOWER versus HIGHER postural sway. For this analysis, a postural sway group (LOWER, HIGHER) by time point (preintervention, post-intervention) mixed analysis of variance (ANOVA) model was created with repeated measures for the time point factor. The results of this ANOVA were considered significant if $P<0.05$, as all subsequent $t$-tests were used to decompose main effects and interactions.

Two supplemental analyses were performed on the data using proportional statistics. First, the proportion of individuals that showed any reduction in postural sway was calculated for all participants, as well as those within the LOWER and HIGHER postural sway groups. Second, the proportion of individuals that showed more than $5 \mathrm{~cm}$ of postural sway reduction was determined for all participants, as well as those within the LOWER and HIGHER postural sway groups. This latter analysis was based on the minimum detectable change
(MDC) statistic that has previously been reported for the BBT. ${ }^{28}$ The MDC reflects the amount of change in the BBT needed to be $90 \%$ confident that a true change in postural sway has occurred due to the Geri-Fit intervention.

\section{Results}

The results of the first-level analysis are shown in Figure 2, which demonstrate a significant $(P<0.05)$ decrease in postural sway across the group of all participants following the Geri-Fit intervention. Despite this positive finding, the second-level analysis, as shown in Figure 3, indicates clear differences $(P<0.05)$ between those individuals who had LOWER versus HIGHER postural sway during preintervention testing. Specifically, there was no significant reduction in postural sway due to Geri-Fit training in those individuals with LOWER postural sway prior to the intervention. In contrast, postural sway was significantly reduced $(P<0.05)$ in the HIGHER postural sway group, who had the highest fall-risk potential during pre-intervention testing. These results were evident despite the LOWER $(n=11)$ and HIGHER ( $n=14)$ postural sway groups showing no significant differences in age $($ LOWER $=74.1$; HIGHER $=74.2)$, height (LOWER $=165.9 \mathrm{~cm}$; HIGHER $=167.4 \mathrm{~cm})$, or body weight (LOWER $=81.0 \mathrm{~kg}$; HIGHER $=86.4 \mathrm{~kg}$ ).

Proportional statistics supported the aforementioned group-based analyses. Figure 4 shows that while $80.8 \%$ of all participants trained using Geri-Fit had a reduction in postural sway, only about half (54.5\%) of those individuals in the LOWER postural sway group had less sway. This was

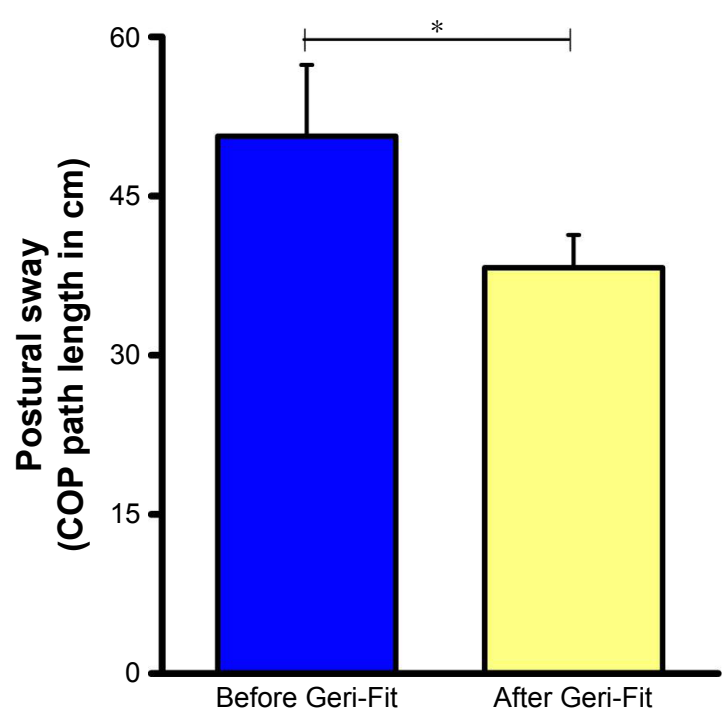

Figure 2 Postural sway was reduced after 90 days of Geri-Fit exercise training. Note: ${ }^{*} P<0.05$.

Abbreviation: COP, center of pressure. 


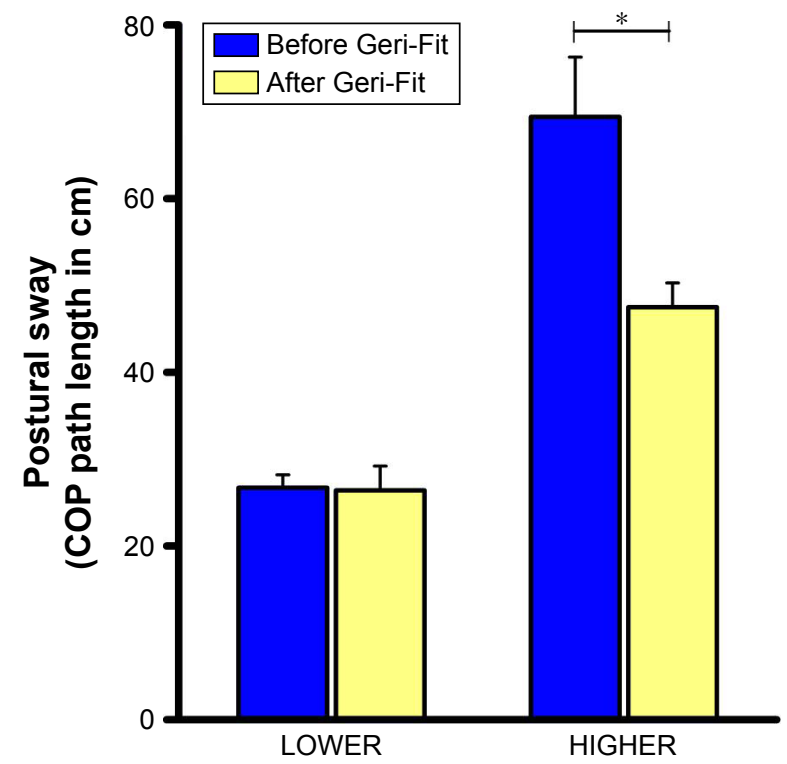

Figure 3 Reductions in postural sway were only evident in those individuals with atypically high postural sway (HIGHER) prior to Geri-Fit training than those with typical postural sway (LOWER).

Note: $* P<0.05$.

Abbreviation: COP, center of pressure.

contrasted by those individuals with HIGHER postural sway, who had the greatest potential (and need) for improvement, where $100 \%$ demonstrated reduced postural sway. Similarly, Figure 5 shows that the total number of individuals who experienced reduced postural sway beyond the MDC for the BBT (ie, $5 \mathrm{~cm}$ ) was $44.0 \%$. However, in the LOWER postural sway group only $9.1 \%$ met these criteria, while in the HIGHER group $71.4 \%$ of those individuals had reduced postural sway beyond the MDC.

\section{Discussion}

The present study determined the effects of Geri-Fit exercise training on postural sway in older adults. Postural sway was determined before and after 90 days of Geri-Fit using a new, low-cost force plate called BTrackS that is specialized for the objective assessment of postural sway and fall risk in older adults. The results showed a significant reduction of postural sway, and presumably fall risk, following Geri-Fit training. This effect was most pronounced for those individuals with HIGHER postural sway pre-intervention than those with LOWER postural sway. Taken together, these findings suggest that BTrackS may provide a practical tool to identify those individuals with HIGHER postural sway, who most likely would benefit from an exercise program like Geri-Fit.

A recent literature review showed postural sway reductions occurred following a number of exercise interventions. ${ }^{19}$ However, the effect of training on postural sway in these studies was somewhat small and inconsistent. ${ }^{20-27}$ The present results suggest that the true benefits of exercise training interventions can only be determined when the pre-intervention postural sway status of participants is considered. In this case, previously reported findings are likely conservative due to the inclusion of individuals with LOWER postural sway who are subject to ceiling effects.

Although sample in this study was relatively small, the effects were large enough to show significant differences across testing time points and between LOWER and HIGHER postural sway groups. This study also lacked a "true" control group of older adults where postural sway assessments were made along a similar time course to those individuals in the present study. To this point, previous research has shown that the BBT is not subject to practice effects in older adults, ${ }^{29,30}$ as evidenced by a high test-retest reliability with intraclass correlation greater than 0.7 .

Even though individuals in the LOWER postural sway group did not typically show reduced postural sway, this does not mean they should be excluded from Geri-Fit training altogether. Indeed, Geri-Fit is a wellness program that has additional benefits beyond the scope of just postural sway and fall-risk mitigation. Further, it should be recognized

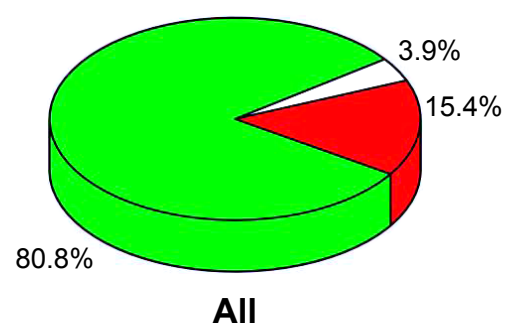

All

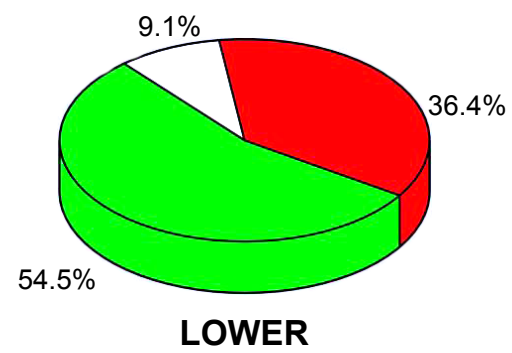

LOWER

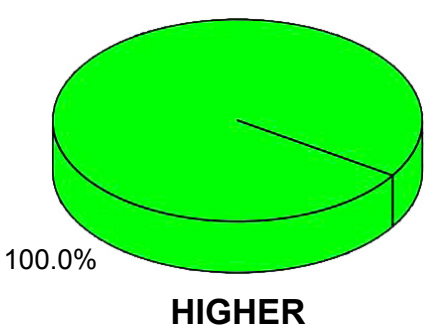

HIGHER

Less postural sway $\square$ Equal postural sway $\square$ More postural sway

Figure 4 The proportion of individuals showing reduced postural sway was larger in the HIGHER than in the LOWER postural sway groups. 


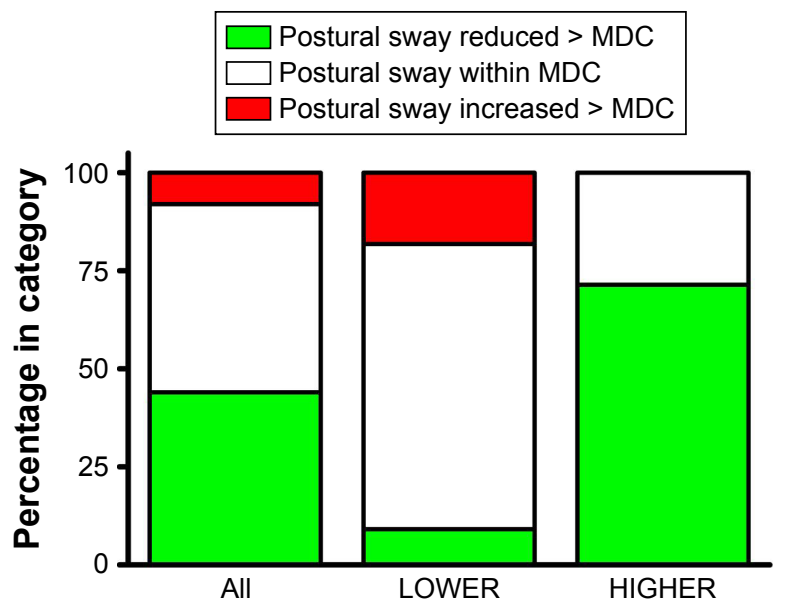

Figure 5 A higher percentage of individuals with HIGHER versus LOWER preintervention postural sway experienced reductions in postural sway greater than the MDC for the BBT.

Abbreviations: MDC, minimum detectable change; BBT, BTrackS Balance Test.

that almost no participants showed a meaningful increase in postural sway that might be associated with poorer balance and/or increased fall risk. To this extent, it seems possible that Geri-Fit training allows for the maintenance of "good" postural sway in those individuals who qualify as being in the LOWER postural sway group.

\section{Conclusion}

The combination of a low-cost force plate like BTrackS and an exercise training intervention like Geri-Fit appears to be an ideal approach for the mitigation of older adult fall risk based on postural sway reductions. Indeed, extensive evidence now exists linking increased postural sway in older adults and a higher likelihood of falls. ${ }^{4-17}$ By screening older adults for fall risk using the BTrackS Assess Balance software criteria, those with HIGHER postural sway can be targeted as candidates who are highly likely to experience reduced postural sway and fall risk with Geri-Fit training. Further, BTrackS testing throughout training, and on a regular basis after the intervention, can serve as an accurate and objective means of monitoring changes in older adult balance.

\section{Acknowledgment}

The authors would like to thank Abby Villacarlos and Carly Graff for assistance with data collection.

\section{Disclosure}

Goble holds an equity stake (ie, stock options) in the parent company for the BTrackS Balance Plate. This conflict of interest was strictly managed by San Diego State University through a mitigation plan that limited his involvement in all primary aspects of data collection and analysis. The other authors report no conflicts of interest in this work.

\section{References}

1. He W, Goodkind D, Kowal P; U.S. Census Bureau, International Population Reports. An aging world: 2015. Washington, DC: U.S. Government Publishing Office; 2016.

2. Ambrose AF, Paul F, Hausdorff JM. Risk factors for falls among older adults: a review of the literature. Maturitas. 2013;75(1):51-61.

3. Centers for Disease Control and Prevention. The State of Aging and Health in America 2013. Atlanta, GA: US Department of Health and Human Services; 2013.

4. Bigelow KE, Berme N. Development of a protocol for improving the clinical utility of posturography as a fall-risk screening tool. J Gerontol A Biol Sci Med Sci. 2011;66(2):228-233.

5. Bird ML, Pittaway JK, Cuisick I, Rattray M, Ahuja KD. Age-related changes in physical fall risk factors: results from a 3 year follow-up of community dwelling older adults in Tasmania, Australia. Int J Environ Res Public Health. 2013;10(11):5989-5997.

6. Boulgarides LK, McGinty SM, Willett JA, Barnes CW. Use of clinical and impairment-based tests to predict falls by community-dwelling older adults. Phys Ther. 2003;83(4):328-339.

7. Hayashibara M, Hagino H, Katagiri H, Okano T, Okada J, Teshima R. Incidence and risk factors of falling in ambulatory patients with rheumatoid arthritis: a prospective 1-year study. Osteoporos Int. 2010;21(11): $1825-1833$.

8. Maki BE, Holliday PJ, Topper AK. A prospective study of postural balance and risk of falling in an ambulatory and independent elderly population. J Gerontol. 1994;49(2):M72-M84.

9. Melzer I, Benjuya N, Kaplanski J. Postural stability in the elderly: a comparison between fallers and non-fallers. Age Ageing. 2004;33(6): 602-607.

10. Melzer I, Kurz I, Oddsson LI. A retrospective analysis of balance control parameters in elderly fallers and non-fallers. Clin Biomech. 2010; 25(10):984-988.

11. Merlo A, Zemp D, Zanda E, et al. Postural stability and history of falls in cognitively able older adults: the Canton Ticino study. Gait Posture. 2012;36(4):662-666.

12. Muir JW, Kiel DP, Hannan M, Magaziner J, Rubin CT. Dynamic parameters of balance which correlate to elderly persons with a history of falls. PLoS One. 2013;8(8):e70566.

13. Pajala S, Era P, Koskenvuo M, Kaprio J, Törmäkangas T, Rantanen T. Force platform balance measures as predictors of indoor and outdoor falls in community-dwelling women aged 63-76 years. J Gerontol A Biol Sci Med Sci. 2008;63(2):171-178.

14. Park JW, Jung M, Kweon M. The mediolateral CoP parameters can differentiate the fallers among the community-dwelling elderly population. J Phys Ther Sci. 2014;26(3):381-384.

15. Swanenburg J, de Bruin ED, Uebelhart D, Mulder T. Falls prediction in elderly people: a 1-year prospective study. Gait Posture. 2010;31(3): 317-321.

16. Taylor ME, Lord SR, Delbaere K, Mikolaizak AS, Close JC. Physiological fall risk factors in cognitively impaired older people: a one-year prospective study. Dement Geriatr Cogn Disord. 2012; 34(3-4):181-189.

17. Thapa PB, Gideon P, Brockman KG, Fought RL, Ray WA. Clinical and biomechanical measures of balance as fall predictors in ambulatory nursing home residents. J Gerontol A Biol Sci Med Sci. 1996; 51(5):M239-M246.

18. O'Connor SM, Baweja HS, Goble DJ. Validating the BTrackS Balance Plate as a low cost alternative for the measurement of swayinduced center of pressure. J Biomech. 2016;49(16):4142-4145.

19. Low DC, Walsh GS, Arkesteijn M. Effectiveness of exercise interventions to improve postural control in older adults: a systematic review and meta-analyses of centre of pressure measurements. Sports Med. 2017;47(1):101-112. 
20. Lai CH, Peng CW, Chen YL, Huang CP, Hsiao YL, Chen SC. Effects of interactive video-game based system exercise on the balance of the elderly. Gait Posture. 2013;37(4):511-515.

21. Lelard T, Doutrellot PL, David P, Ahmaidi S. Effects of a 12-week Tai Chi Chuan program versus a balance training program on postural control and walking ability in older people. Arch Phys Med Rehabil. 2010;91(1):9-14.

22. Nagai K, Yamada M, Tanaka B, et al. Effects of balance training on muscle coactivation during postural control in older adults: a randomized controlled trial. J Gerontol A Biol Sci Med Sci. 2012;67(8):882-889.

23. Ni M, Mooney K, Richards L, et al. Comparative impacts of Tai Chi, balance training, and a specially-designed yoga program on balance in older fallers. Arch Phys Med Rehabil. 2014;95(9):1620-1628.e30.

24. Pluchino A, Lee SY, Asfour S, Roos BA, Signorile JF. Pilot study comparing changes in postural control after training using a video game balance board program and 2 standard activity-based balance intervention programs. Arch Phys Med Rehabil. 2012;93(7):1138-1146.

25. Song CH, Petrofsky JS, Lee SW, Lee KJ, Yim JE. Effects of an exercise program on balance and trunk proprioception in older adults with diabetic neuropathies. Diabetes Technol Ther. 2011;13(8):803-811.
26. Lee SW, Song CH. Virtual reality exercise improves balance of elderly persons with type 2 diabetes: a randomized controlled trial. J Phys Ther Sci. 2012;24:261-265.

27. Nicholson VP, McKean MR, Burkett BJ. Twelve weeks of BodyBalance ${ }^{\sqrt{R}}$ training improved balance and functional task performance in middleaged and older adults. Clin Interv Aging. 2014;9:1895-1904.

28. Goble DJ, Manyak KA, Abdenour TE, Rauh MJ, Baweja HS. An initial evaluation of the BTrackS Balance Plate and Sports Balance software for concussion diagnosis. Int J Sports Phys Ther. 2016;11(2):149-155.

29. Lin D, Seol H, Nussbaum MA, Madigan ML. Reliability of COP-based postural sway measures and age-related differences. Gait Posture. 2008; 28(2):337-342.

30. Golriz S, Hebert JJ, Foreman KB, Walker BF. The reliability of a portable clinical force plate used for the assessment of static postural control: repeated measures reliability study. Chiropr Man Therap. 2012; 20(1): 14 .
Clinical Interventions in Aging

\section{Publish your work in this journal}

Clinical Interventions in Aging is an international, peer-reviewed journal focusing on evidence-based reports on the value or lack thereof of treatments intended to prevent or delay the onset of maladaptive correlates of aging in human beings. This journal is indexed on PubMed Central, MedLine,

\section{Dovepress}

CAS, Scopus and the Elsevier Bibliographic databases. The manuscript management system is completely online and includes a very quick and fair peer-review system, which is all easy to use. Visit http://www.dovepress. $\mathrm{com} /$ testimonials.php to read real quotes from published authors. 